\title{
Null-field integral equations and their applications
}

\author{
J.-T. Chen \& P.-Y. Chen \\ Department of Harbor and River Engineering, \\ National Taiwan Ocean University, Keelung, Taiwan
}

\begin{abstract}
In this paper, a null-field equation approach is proposed to deal with boundary value problems containing circular boundaries. The mathematical tools, degenerate kernels and Fourier series, are utilized in the null-field integral formulation. Although we employ the null field equations, we can exactly collocate the point on the real boundary. Thus, the singularity is novelly avoided since the kernel is expressed in a degenerate form. Five gains of well-posed model, singularity free, boundary layer effect free, exponential convergence and mesh-free approach are achieved. To demonstrate the validity of the present formulation, some applications are considered: (1) torsion problem, (2) bending problem, (3) SH wave impinging successive canyons. It is found that previous results by other investigators are not consistent with ours. After comparing with other independent solutions, the accuracy and efficiency of our approach is acceptable.
\end{abstract}

Keywords: null-field integral equation, degenerate kernel, Fourier series.

\section{Introduction}

Engineering problems can be formulated as mathematical models. In order to solve the boundary value problems, researchers and engineers have paid more attention on the development of boundary integral equation method (BIEM), boundary element method (BEM) and meshless method than domain type methods, finite element method (FEM) and finite difference method (FDM). Among various numerical methods, BEM is one of the most popular numerical approaches for solving boundary value problems. Although BEM has been 
involved as an alternative numerical method for solving engineering problems, five critical issues are of concern.

(1) Treatment of singularity and hypersingularity. It is well known that BEM are based on the use of fundamental solutions to solve partial differential equations. These solutions are two-point functions which are singular as the source and field points coincide. Several regularizations for hypersingularity were offered to handle it in direct and indirect ways. In the present approach, we employed the degenerate kernel to represent the fundamental solution for problems with circular boundaries. The singularity and hypersingularity disappeared in the boundary integral equation after describing the potential into two parts.

(2) Boundary-layer effect. Boundary-layer effect in BEM has received attention in the recent years. In real applications, data near boundary can be smoothened since maximum principle exists for potential problems. Nevertheless, it also deserves study to know how to manipulate the nearly singular integrals in applied mathematics. How to eliminate the boundarylayer effect in BEM is vital for researchers.

(3) Convergence rate. Regarding to constant, linear and quadratic elements, the discretization scheme does not take the special geometry into consideration. It leads to the slow convergence rate. For example, Fourier series is suitable for boundary densities on circular boundaries. Although previous researchers have employed the Fourier series expansion, no one has ever introduced the degenerate kernel in boundary integral equations to tackle their problems. Mathematicians have proved that the exponential convergence instead of the algebraic convergence in the BEM can be achieved by using the degenerate kernel and Fourier expansion.

(4) Ill-posed model. Null-field approach or fictitious BEM free of calculating the singular and hypersingular integrals yields an ill-conditioned system. To approach the fictitious boundary to the real boundary or to move the nullfield point to the real boundary can make the system well-posed. However, singularity appears in the meantime. We may wonder is it possible to push the null-field point on the real boundary but free of facing the singular and hypersingular integrals. The answer is yes and can be found in this paper.

(5) Mesh on boundary is still necessary.

The five issues, singularity free, the suppression of boundary-layer effect, exponential convergence, well-posed model and mesh-free will be examined in this paper.

Engineering problems with circular boundaries are often encountered, e.g. missiles, aircraft, naval architecture, etc., either to reduce the weight of the whole structure or to increase the range of inspection as well as piping purposes. Analytical approach using bi-polar coordinate [1] was developed for two-hole problems. Complex variable techniques were also employed for the annular case. For a problem with several holes, many numerical methods, e.g. finite element method (FEM) and boundary element method (BEM) were resorted to solve. To develop a systematic approach for engineering problems with circular boundaries is not trivial. 
Null-field integral equation approach is used widely for obtaining the numerical solutions of engineering problems. Various names, e.g. T-matrix method [2] and extended boundary condition method (EBCM) [3], have been coined. A crucial advantage of this method consists in the fact that the influence matrix can be computed easily. Although many works for acoustic and water wave problems have been done, we focus on the solid mechanics here.

In this paper, we review the recent development of the null-field integral equation approach [4-9] for boundary value problems (BVPs) with circular boundaries. The key idea is the expansion of kernel functions and boundary densities in the null-field integral equations. Vector decomposition technique using the adaptive observer system is required for nonfocal cases. Applications to the Laplace and Helmholtz problems are addressed. Several examples were demonstrated to see the validity of the new formulation.

\section{Null-field integral equation approach for boundary value problems}

Suppose there are $N$ randomly distributed circular boundaries bounded to the domain $D$ and enclosed with the boundary, $B_{k}(k=0,1,2, \cdots, N)$ as shown in Figure 1. We define

$$
B=\bigcup_{k=0}^{N} B_{k} .
$$

In mathematical physics, boundary value problems can be modelled by the governing equation,

$$
L u(x)=0, x \in D,
$$

where $L$ may be the Laplace, Helmholtz, biharmonic or biHelmholtz operator, $u(\mathrm{x})$ is the potential function and $D$ is the domain of interest. For the 2-D Laplace and Helmholtz problems, the integral equation for the domain point can be derived from the third Green's identity, we have

$$
\begin{aligned}
& 2 \pi u(\mathrm{~s})=\int_{B} T(\mathrm{~s}, \mathrm{x}) u(\mathrm{~s}) d B(\mathrm{~s})-\int_{B} U(\mathrm{~s}, \mathrm{x}) t(\mathrm{~s}) d B(\mathrm{~s}), \mathrm{x} \in D, \\
& 2 \pi \frac{\partial u(\mathrm{x})}{\partial \mathrm{n}_{\mathrm{x}}}=\int_{B} M(\mathrm{~s}, \mathrm{x}) u(\mathrm{~s}) d B(\mathrm{~s})-\int_{B} L(\mathrm{~s}, \mathrm{x}) t(\mathrm{~s}) d B(\mathrm{~s}), \mathrm{x} \in D,
\end{aligned}
$$

where $\mathrm{s}$ and $\mathrm{x}$ are the source and field points, respectively, $t=\partial u / \partial \mathrm{n}, B$ is the boundary, $\mathrm{n}_{\mathrm{x}}$ denotes the outward normal vector at the field point $\mathrm{x}$ and the kernel function $U(\mathrm{~s}, \mathrm{x})$, is the fundamental solution, and the other kernel functions, $T(\mathrm{~s}, \mathrm{x}), L(\mathrm{~s}, \mathrm{x})$ and $M(\mathrm{~s}, \mathrm{x})$, are defined in the dual boundary integral method (BIEM) [9]. It is noted that more potentials are needed in eqns (3) and (4) for biharmonic and biHelmholtz cases. By moving the field point to the boundary, the eqns (3) and (4) reduce to 


$$
\begin{aligned}
& \pi u(\mathrm{x})=C . P . V . \int_{B} T(\mathrm{~s}, \mathrm{x}) u(\mathrm{~s}) d B(\mathrm{~s})-R . P . V . \int_{B} U(\mathrm{~s}, \mathrm{x}) t(\mathrm{~s}) d B(\mathrm{~s}), \mathrm{x} \in B \\
& \pi \frac{\partial u(\mathrm{x})}{\partial \mathrm{n}_{\mathrm{x}}}=H . P . V . \int_{B} M(\mathrm{~s}, \mathrm{x}) u(\mathrm{~s}) d B(\mathrm{~s})-C . P . V \cdot \int_{B} L(\mathrm{~s}, \mathrm{x}) t(\mathrm{~s}) d B(\mathrm{~s}), \mathrm{x} \in B
\end{aligned}
$$

where C.P.V., R.P.V. and H.P.V. denote the Cauchy principal value, Riemann principal value and Hadamard principal value, respectively. By collocating the field point $\mathrm{x}$ outside the domain (including the boundary), the null-field integral equations yield

$$
\begin{gathered}
0=\int_{B} T(\mathrm{~s}, \mathrm{x}) u(\mathrm{~s}) d B(\mathrm{~s})-\int_{B} U(\mathrm{~s}, \mathrm{x}) t(\mathrm{~s}) d B(\mathrm{~s}), \mathrm{x} \in D^{c} \cup B \\
0=\int_{B} M(\mathrm{~s}, \mathrm{x}) u(\mathrm{~s}) d B(\mathrm{~s})-\int_{B} L(\mathrm{~s}, \mathrm{x}) t(\mathrm{~s}) d B(\mathrm{~s}), \mathrm{x} \in D^{c} \cup B
\end{gathered}
$$

by choosing appropriate forms of degenerate kernels, where $D^{c}$ is the complementary domain.
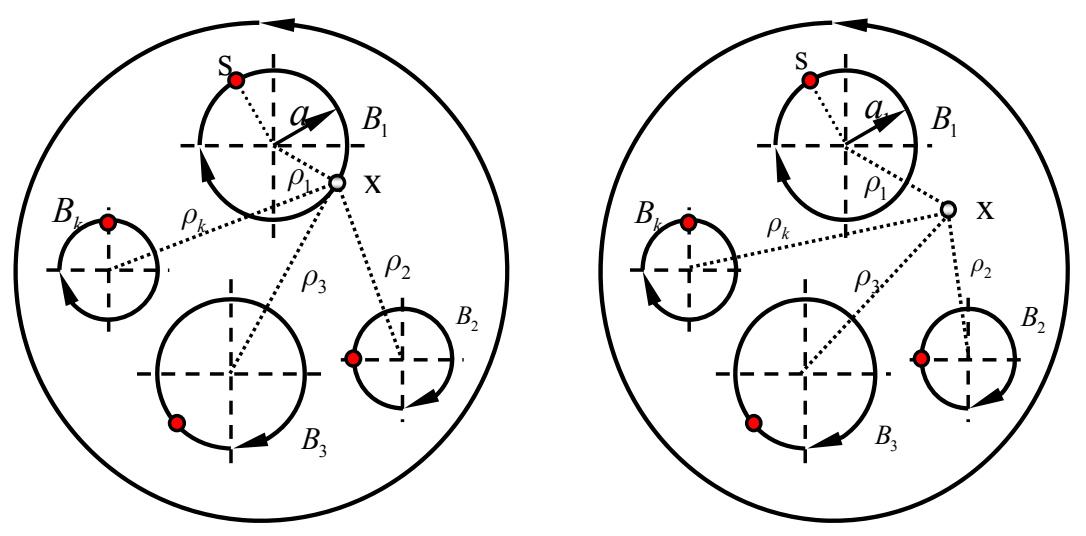

Figure 1: Sketch of null-field and domain points in conjunction with the adaptive observer system (left: collocation on the boundary point, right: collocation on the interior point).

\section{Expansions of the fundamental solution and boundary density}

Instead of directly calculating the C.P.V., R.P.V. and H.P.V. in eqns (5) and (6), we obtain the linear algebraic system from the null-field integral equations of eqns (7) and (8) through the kernel expansion.

Based on the separable property, the kernel function $U(\mathrm{~s}, \mathrm{x})$ can be expanded into the separable form by dividing the source and field points: 


$$
U(\mathrm{~s}, \mathrm{x})=\left\{\begin{array}{l}
U^{i}(\mathrm{~s}, \mathrm{x})=\sum_{j=1}^{\infty} A_{j}(\mathrm{~s}) B_{j}(\mathrm{x}),|\mathrm{s}| \geq|\mathrm{x}|, \\
U^{e}(\mathrm{~s}, \mathrm{x})=\sum_{j=1}^{\infty} A_{j}(\mathrm{x}) B_{j}(\mathrm{~s}),|\mathrm{x}| \geq|\mathrm{s}|,
\end{array}\right.
$$

where the $A(\mathrm{x})$ and $B(\mathrm{x})$ can be found for the Laplace [4-8], Helmholtz [9], biharmonic [5] and biHelmholtz operators and the superscripts " $i$ " and " $e$ " denote the interior $(|\mathrm{s}| \geq|\mathrm{x}|)$ and exterior $(|\mathrm{s}|<|\mathrm{x}|)$ cases, respectively. To classify the interior and exterior regions, Figure 2 shows for one-, two- and three-dimensional cases. For the degenerate forms of $T, L$ and $M$ kernels, they can be derived according to their definitions. We apply the Fourier series expansions to approximate the potential $u$ and its normal derivative $t$ on the $B_{k}$ circular boundary

$$
\begin{aligned}
& u\left(\mathrm{~s}_{k}\right)=a_{0}^{k}+\sum_{n=1}^{\infty}\left(a_{n}^{k} \cos n \theta_{k}+b_{n}^{k} \sin n \theta_{k}\right) \\
& t\left(\mathrm{~s}_{k}\right)=p_{0}^{k}+\sum_{n=1}^{\infty}\left(p_{n}^{k} \cos n \theta_{k}+q_{n}^{k} \sin n \theta_{k}\right)
\end{aligned}
$$

where $a_{n}^{k}, b_{n}^{k}, p_{n}^{k}$ and $q_{n}^{k}(k=0,1,2, \cdots, N)$ are the Fourier coefficients and $\theta_{k}$ is the polar angle measured with respect to the $x$-direction.

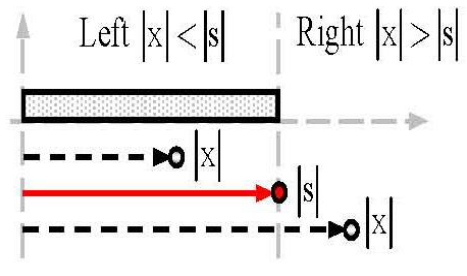

1D

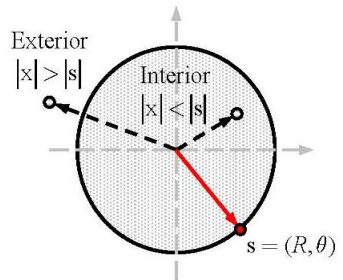

2D

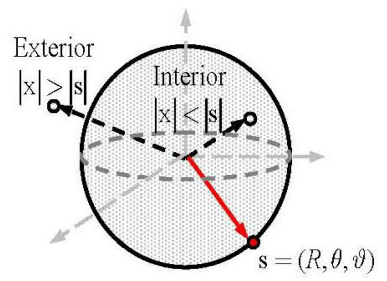

3D

Figure 2: The degenerate kernel for the one-, two- and three-dimensional problems.

After collocating the null-field points in the null-field integral equation of eqn (7), the boundary integrals through all the circular contours are required. It is worth noting that the origin of the observer system is located on the center of the 
corresponding circle under integration to entirely utilize the geometry of circular boundary for the expansion of degenerate kernels and boundary densities. Figure 1 shows the boundary integration for the circular boundaries in the adaptive observer system. By collocating the null-field point $\mathrm{x}_{k}$ exactly on the $k$ th circular boundary for eqn (7) in Figure 1, we have

$$
[U]\{t\}=[T]\{u\}
$$

where $[U]$ and $[T]$ are the influence matrices with a dimension of $(N+1)(2 m+1)$ by $(N+1)(2 m+1),\{u\}$ and $\{t\}$ denote the column vectors of Fourier coefficients with a dimension of $(N+1)(2 m+1)$ by 1 in which $m$ indicates the truncated terms of Fourier series. For the circular-inclusion problem, multi-domain approach by taking the free body of each interface between the matrix and inclusions should be introduced. Therefore, an exterior problem for the matrix and several interior problems for each inclusion are needed to be solved by employing the null-field approach. The continuity of displacement and equilibrium of traction should be considered on the interface between the matrix and inclusions $[8,9]$. Then, the resulted linear algebraic system is obtained. After the boundary unknowns are obtained, the field potential can be easily obtained according to eqn (3).

\section{Illustrative examples}

\section{Case 1: A circular bar with multiple circular holes under torsion (Laplace equation)}

A circular bar with multiple equal circular holes removed is under torque at the end $[10,11]$. Table 1 shows the comparison of the torsional rigidities $G$ of three cases with different geometries of circular holes. The present solutions show improvement over Ling's results [10] in every case. The discrepancy in the second example in Table 1 may ascribe to the Ling's lengthy calculation in error as pointed out by Caulk [11].

Table 1: $\quad$ Torsional rigidity in Ling's examples [11].

\begin{tabular}{|c|c|c|c|}
\hline \multicolumn{4}{|l|}{ Case } \\
\hline & $a / a_{0}=2 / 7, b / a_{0}=3 / 7$ & $\begin{array}{l}c / a_{0}=1 / 5, a / a_{0}=1 / 5, \\
b / a_{0}=3 / 5\end{array}$ & $\begin{array}{l}c / a_{0}=1 / 5, a / a_{0}=1 / 5, \\
b / a_{0}=3 / 5\end{array}$ \\
\hline Caulk [11] & 0.8713 & 0.8732 & 0.7261 \\
\hline Ling's results [10] & 0.8809 & 0.8093 & 0.7305 \\
\hline $\begin{array}{l}\text { Present method } \\
(m=10)\end{array}$ & 0.8712 & 0.8732 & 0.7244 \\
\hline
\end{tabular}




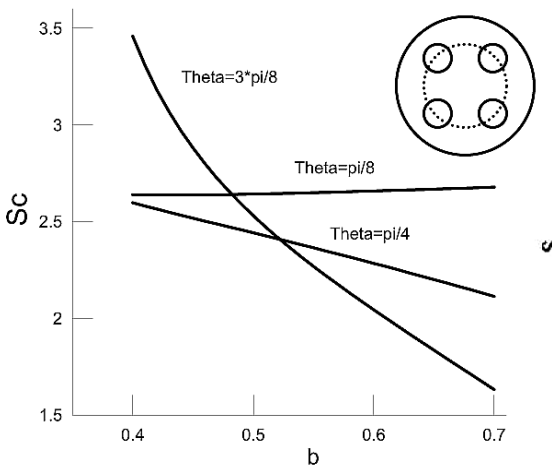

(a)

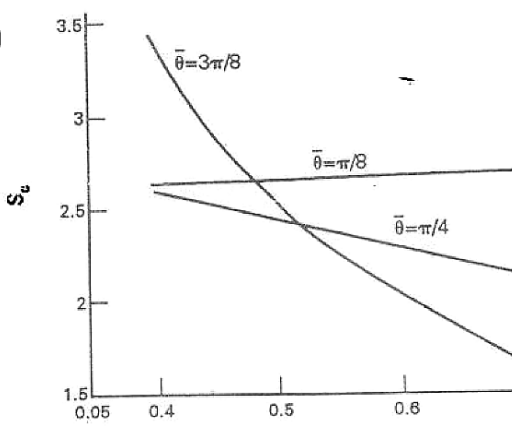

(b)

Figure 3: Stress concentration versus $b$ for $a=0.12, R=1.0$ and three different values of $\bar{\theta}=\pi / 8, \bar{\theta}=\pi / 4$ and $\bar{\theta}=3 \pi / 8$. (a) Sc at the point B (present method); (b) Sc at the point B (Naghdi's result [12]).

Case 2: A circular beam with four circular holes under bending (Laplace equation)

Naghdi [12] and Bird and Steele [13] both calculated the stress concentration for the four equal-sized circular holes problem under bending. Bird and Steele [13] stated that the deviation by the Naghdi's data is $11 \%$. The grounds for this discrepancy were not identified in their paper. Our numerical results agree well with the Naghdi's data as shown in Figure 3.

Case 3: Two canyons subject to the incident SH-wave (Helmholtz equation-a half plane problem)

Tsaur et al. [14] and Fang [15] both calculated the response of two canyons subject to the incident SH-wave. Tsaur et al. [14] pointed out that the error of Fang [15] is due to wrong use of orthogonal properties. Good agreement is made after comparing with the results of Tsaur et al. [14] as shown in Figure 4.

\section{Conclusions}

A semi-analytical approach was proposed for solving BVPs with circular boundaries. Some recent results were reviewed. The key idea is that we can collocate on the real boundary although we employ the concept of null field equations. Not only the singularity is transformed to the series sum but also the boundary-layer effect is eliminated. In order to verify the formulation, applications to the Laplace and Helmholtz problems were done. Five gains of well-posed model, singularity free, boundary layer effect free, exponential convergence and mesh-free approach were achieved. 
96 Boundary Elements and Other Mesh Reduction Methods XXIX

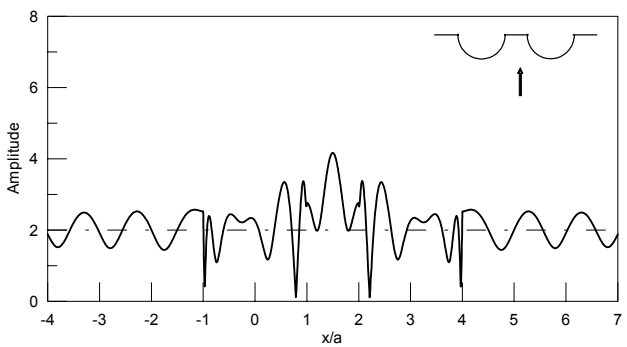

(a) $\gamma=0^{\circ}$

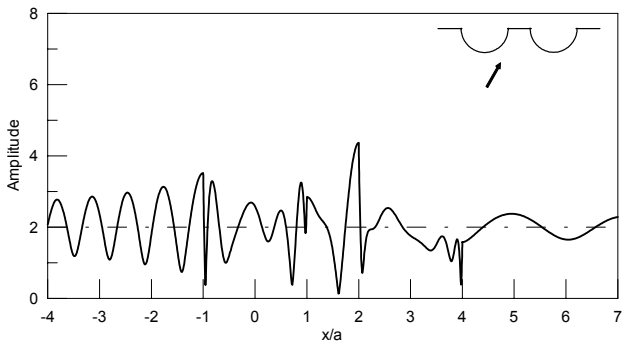

(b) $\gamma=30^{\circ}$

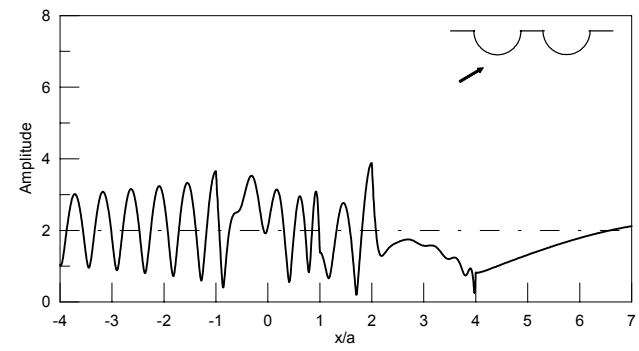

(c) $\gamma=60^{\circ}$

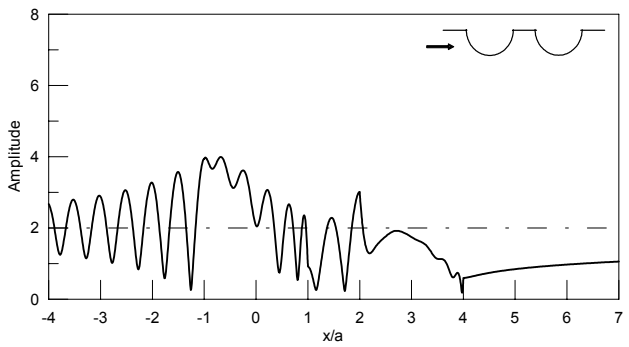

(d) $\gamma=90^{\circ}$

Figure 4: $\quad$ Surface displacements of two canyons problem $\left(\mu^{I} / \mu^{M}=10^{-8}\right.$ and $\eta=2$ ). 


\section{References}

[1] Lebedev, N.N., Skalskaya, I.P. \& Uflyand, Y.S., Worked problem in applied mathematics. Dover Publications: New York, 1979.

[2] Waterman, P.C., Matrix formulation of electromagnetic scattering. Proc. IEEE, 53, pp. 805-812, 1965.

[3] Doicu, A. \& Wriedt, T., Extended boundary condition method with multiple sources located in the complex plane. Optics Communications, 139, pp. 85-91, 1997.

[4] Chen, J.T., Shen, W.C. \& Wu, A.C., Null-field integral equations for stress field around circular holes under anti-plane shear. Engineering Analysis with Boundary Elements, 30, pp. 205-217, 2005.

[5] Chen, J.T., Hsiao, C.C. \& Leu, S.Y., Null-field integral equation approach for plate problems with circular holes. ASME Journal of Applied Mechanics, 73, pp. 679-693, 2006.

[6] Chen, J.T., Shen, W.C. and Chen, P.Y., Analysis of circular torsion bar with circular holes using null-field approach. Computer Modelling in Engineering Science, 12, pp. 109-119, 2006.

[7] Chen, J.T. \& Wu, A.C., Null-field approach for multi-inclusion problem under anti-plane shears. ASME Journal of Applied Mechanics, Accepted, 2007.

[8] Chen, J.T. \& Wu, A.C., Null-field approach for piezoelectricity problems with arbitrary circular inclusions. Engineering Analysis with Boundary Elements, Accepted, 2006.

[9] Chen, J.T., Null filed integral equation approach for boundary vale problems with circular boundaries, Keynote lecture of ICCES 2005, India, 2005.

[10] Ling, C.B., Torsion of a circular tube with longitudinal circular holes. Quarterly of Applied Mathematics, 5, pp. 168-181, 1947.

[11] Caulk, D.A., Analysis of elastic torsion in a bar with circular holes by a special boundary integral method. ASME Journal of Applied Mechanics, 50, pp. 101-108, 1983.

[12] Naghdi, A.K., Bending of a perforated circular cylindrical cantilever. International Journal of Solids and Structures, 28, pp. 739-749, 1991.

[13] Bird, M.D. \& Steele, C.R., A solution procedure for Laplace's equation on multiply connected circular domains. ASME Journal of Applied Mechanics, 59, pp. 398-404, 1992.

[14] Tsaur, D.H., Chang, K.H. \& Lin, J.G., Response of multiple semi-circular cylindrical vanyons subject to plane SH-waves, Asia Pacific Review of Engineering Science and Technology, 2(2), pp. 251-266, 2004. (in Chinese)

[15] Fang, Y.G., Scattering of plane SH-waves by multiple circular-arc valleys at the two-dimensional surface of the earth. Earthquake Engineering and Engineering Vibration, 15(1), pp. 85-91, 1995. (in Chinese) 